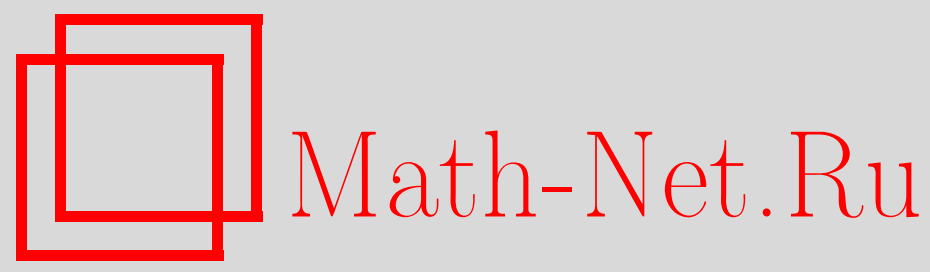

А. М. Попов, Условия потенциальности Гельмгольца для систем дифференциально-разностных уравнений, Матем. заметки, 1998, том 64, выпуск 3, 437-442

DOI: https://doi.org/10.4213/mzm1415

Использование Общероссийского математического портала Math-Net.Ru подразумевает, что вы прочитали и согласны с пользовательским соглашением http://www . mathnet.ru/rus/agreement

Параметры загрузки:

IP : 54.198 .55 .26

26 апреля 2023 г., $16: 52: 45$

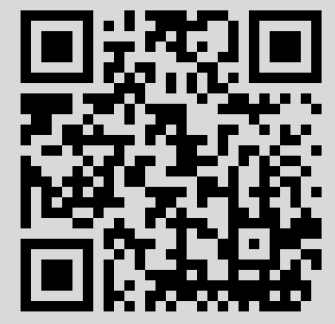




\section{УСЛОВИЯ ПОТЕНЦИАЛЬНОСТИ ГЕЛЬМГОЛЬЦА ДЛЯ СИСТЕМ ДИФФЕРЕНЦИАЛЬНО-РАЗНОСТНЫХ УРАВНЕНИЙ}

\section{А. М. Попов}

Для операторов, соответствующих системам дифференциально-разностных уравнений $\mu$-го порядка нейтрального типа, получены необходимые и достаточные условия потенциальности относительно классической билинейной формы. Полученные результаты применяются к квазилинейным системам первого порядка.

Библиография: 14 названий.

1. Система $\mu$-го порядка. Условия потенциальности операторов, соответствующих обькновенньп дифференциальньп уравнениям, рассматривались многими авторами (см. [1]-[6]). В наиболее полном объеме эти условия потенциальности приведены в книге [6]. Там же, а также в монографиях В. М. Филиппова [7] и В. М. Савчина [8] имеется обширная библиография по вариационньм методам решений уравнений. Впервые необходимые условия потенциальности для обькновенных дифференциальных уравнений были получены Гельмгольцем (см. [1] , [2]), поэтому подобные условия потенциальности для других классов уравнений в современной литературе принято назьвать условиями потенциальности Гельмгольца.

В настоящей заметке рассматриваются системы дифференциально-разностных уравнений нейтрального типа. Для операторов, соответствуюших системам таких уравнений $\mu$-го порядка, получены необходимые и достаточные условия потенциальности, которые затем применяются для систем уравнений первого порядка.

Рассмотрим систему дифференциально-разностных уравнений нейтрального типа

$$
F_{i}\left(t, u^{(m)}(t+\vartheta \tau)\right)=0
$$

где $i=1, \ldots, n, m=0, \ldots, \mu, \vartheta=-1,0,1, \tau=$ const $>0, u=\left(u_{1}, u_{2}, \ldots, u_{n}\right), F_{i} \in C$.

Введем оператор $N$

$$
N(u)=\left(F_{1}, F_{2}, \ldots, F_{n}\right)
$$

Система (1) эквивалентна операторному уравнению

$$
N(u)=0_{V}
$$

где $0_{V}-$ нулевой элемент в $V$. 
Обозначим через $U=Q^{\mu}$ множество непрерьвных $\mu$ раз кусочно-дифференцируемьх вектор-функций (назьваемых в дальнейшем просто функциями) при $t \in\left[t_{1}-\tau, t_{2}+\tau\right]$, $t_{2}-t_{1}>2 \tau$.

Зададим область определения рассматриваемого оператора $N$ (2) равенством

$$
\begin{aligned}
D(N)=\left\{u \in U: u^{(l)}(t)\right. & =\varphi_{1}^{(l)}(t), t \in E_{1}=\left[t_{1}-\tau, t_{1}+\tau\right], \\
u^{(l)}(t) & \left.=\varphi_{2}^{(l)}(t), t \in E_{2}=\left[t_{2}-\tau, t_{2}+\tau\right]\right\},
\end{aligned}
$$

где $\varphi_{1}$ и $\varphi_{2}-$ заданные функции, $l=0, \ldots,[\mu / 2],[\cdot]-$ целая часть.

Пусть $N: D(N) \rightarrow V$. На множестве $V \times U$ зададим локальную билинейную форму $\langle\cdot, \cdot\rangle: V \times U \rightarrow \mathbb{R}$ равенством

$$
\langle\nu, g\rangle=\int_{t_{1}}^{t_{2}} \nu(t) g(t) d t,
$$

где $\nu(t) g(t)=\nu_{i}(t) g_{i}(t)$. Здесь и далее по повторяющимся индексам сомножителей производится суммирование от 1 до $n$.

Цель настоящей работы - нахождение условий потенциальности операторов, соответствующих дифференциально-разностным уравнениям. В связи с этим приведем определение потенциального оператора [6, с. 10].

Оператор $N$ назьвается потенииальным относительно билинейной формы (5), если существует дифференцируемый по Гато функционал $f_{N}: D\left(f_{N}\right) \equiv D(N) \rightarrow \mathbb{R}$ такой, что

$$
\delta f_{N}[u, h]=\langle N(u), h\rangle, \quad u \in D(N) .
$$

Теорема 1. Пусть $F_{i} \in C^{\mu+1}$. Для того чтобы оператор $N$ (2) уравнения (1) (или, что то жее самое, уравнения (3)) был потенииальным в области (4) относительно билинейной формы (5), необходимо и достаточно выполнения равенств

$$
\begin{gathered}
{\left[\frac{\partial F_{i}}{\partial u_{j}(t-\tau)}+\sum_{m=1}^{\mu}(-1)^{m} \frac{d^{m}}{d t^{m}} \frac{\partial F_{i}}{\partial u_{j}^{(m)}(t-\tau)}\right]_{t \rightarrow t+\tau}=\frac{\partial F_{j}}{\partial u_{i}(t+\tau)}} \\
\frac{\partial F_{i}}{\partial u_{j}(t)}+\sum_{m=1}^{\mu}(-1)^{m} \frac{d^{m}}{d t^{m}} \frac{\partial F_{i}}{\partial u_{j}^{(m)}(t)}=\frac{\partial F_{j}}{\partial u_{i}(t)} \\
{\left[\frac{\partial F_{i}}{\partial u_{j}(t+\tau)}+\sum_{m=1}^{\mu}(-1)^{m} \frac{d^{m}}{d t^{m}} \frac{\partial F_{i}}{\partial u_{j}^{(m)}(t+\tau)}\right]_{t \rightarrow t-\tau}=\frac{\partial F_{j}}{\partial u_{i}(t-\tau)}} \\
\sum_{m=k}^{\mu}(-1)^{m}\left(\begin{array}{c}
m \\
k
\end{array}\right)\left[\frac{d^{m-k}}{d t^{m-k}} \frac{\partial F_{i}}{\partial u_{j}^{(m)}(t-\tau)}\right]_{t \rightarrow t+\tau}=\frac{\partial F_{j}}{\partial u_{i}^{(k)}(t+\tau)} \\
\sum_{m=k}^{\mu}(-1)^{m}\left(\begin{array}{c}
m \\
k
\end{array}\right) \frac{d^{m-k}}{d t^{m-k}} \frac{\partial F_{i}}{\partial u_{j}^{(m)}(t)}=\frac{\partial F_{j}}{\partial u_{i}^{(k)}(t)}, \\
\sum_{m=k}^{\mu}(-1)^{m}\left(\begin{array}{c}
m \\
k
\end{array}\right)\left[\frac{d^{m-k}}{d t^{m-k}} \frac{\partial F_{i}}{\partial u_{j}^{(m)}(t+\tau)}\right]_{t \rightarrow t-\tau}=\frac{\partial F_{j}}{\partial u_{i}^{(k)}(t-\tau)}
\end{gathered}
$$

әде $i, j=1, \ldots, n, k=1, \ldots, \mu$, а запись $[\Phi]_{t \rightarrow t+\tau}\left(\right.$ или $\left.[\Phi]_{t \rightarrow t-\tau}\right)$ означает, что в выражении для $\Phi$ вместо $t$ следует подставить $t+\tau($ или $t-\tau)$. 
ДокАЗАтЕльство. Для доказательства теоремы воспользуемся теоремой Вольтерра-Кернера-Вайнберга (см. [6, с. 15] и [9, с. 66]).

Если оператор $N$ дифференцируем по Гато в каждой точке $u \in U$, причем дифференциал $D N(u, h)$ непрерьвен в каждой точке $u \in U$, то для потенциальности $N$ необходимо и достаточно вьполнения равенства

$$
\left\langle N_{u}^{\prime} h, g\right\rangle=\left\langle N_{u}^{\prime} g, h\right\rangle, \quad u \in U, \quad g, h \in D\left(N_{u}^{\prime}\right)
$$

где

$$
D\left(N_{u}^{\prime}\right)=\left\{h \in D(N): h=0, t \in E_{1} \cup E_{2}\right\}
$$

Очевидно, что условия дифференцируемости по Гато оператора $N$ и непрерьвности дифференциала $D N(u, h)$ выполняются. Проверим условие симметричности (7). Для этого сначала находим $\left\langle N_{u}^{\prime} h, g\right\rangle$. Применяя интегрирование по частям, соответствующие замены переменной интегрирования, формулу Лейбница и условия (8) для $h$ и $g$, получим

$$
\begin{aligned}
\left\langle N_{u}^{\prime} h, g\right\rangle=\int_{t_{1}}^{t_{2}} \sum_{m=0}^{\mu}\left(\frac{\partial F_{i}}{\partial u_{j}^{(m)}(t-\tau)} h_{j}^{(m)}(t-\tau)+\frac{\partial F_{i}}{\partial u_{j}^{(m)}(t)} h_{j}^{(m)}(t)\right. \\
\left.\quad+\frac{\partial F_{i}}{\partial u_{j}^{(m)}(t+\tau)} h_{j}^{(m)}(t+\tau)\right) g_{i}(t) d t \\
=\int_{t_{1}}^{t_{2}}\left(\left[\frac{\partial F_{i}}{\partial u_{j}(t-\tau)}\right]_{t \rightarrow t+\tau} g_{i}(t+\tau)+\frac{\partial F_{i}}{\partial u_{j}(t)} g_{i}(t)+\left[\frac{\partial F_{i}}{\partial u_{j}(t+\tau)}\right]_{t \rightarrow t-\tau} g_{i}(t-\tau)\right. \\
\quad+\sum_{k=1}^{\mu} \sum_{m=k}^{\mu}(-1)^{m}\left(\begin{array}{c}
m \\
k
\end{array}\right)\left(\left[\frac{d^{m-k}}{d t^{m-k}} \frac{\partial F_{i}}{\partial u_{j}(t-\tau)}\right]_{t \rightarrow t+\tau} g_{i}^{(k)}(t+\tau)\right. \\
\left.\left.\quad+\frac{d^{m-k}}{d t^{m-k}} \frac{\partial F_{i}}{\partial u_{j}(t)} g_{i}^{(k)}(t)+\left[\frac{d^{m-k}}{d t^{m-k}} \frac{\partial F_{i}}{\partial u_{j}(t+\tau)}\right]_{t \rightarrow t-\tau} g_{i}^{(k)}(t-\tau)\right)\right) h_{j}(t) d t .
\end{aligned}
$$

Теперь находим

$$
\begin{aligned}
& \left\langle N_{u}^{\prime} h, g\right\rangle=\int_{t_{1}}^{t_{2}}\left(\frac{\partial F_{j}}{\partial u_{i}^{(m)}(t-\tau)} g_{i}(t-\tau)+\frac{\partial F_{j}}{\partial u_{i}^{(m)}(t)} g_{i}(t)+\frac{\partial F_{j}}{\partial u_{i}(t+\tau)} g_{i}(t+\tau)\right. \\
& \left.\quad+\sum_{k=1}^{\mu}\left(\frac{\partial F_{j}}{\partial u_{i}^{(k)}(t-\tau)} g_{i}^{(k)}(t-\tau)+\frac{\partial F_{j}}{\partial u_{i}^{(k)}(t)} g_{i}^{(k)}(t)+\frac{\partial F_{j}}{\partial u_{i}^{(k)}(t+\tau)} g_{i}^{(k)}(t+\tau)\right)\right) h_{j}(t) d t .
\end{aligned}
$$

Сравнивая выражения (9) и (10) и принимая во внимание произвольность допустимых функций $h(t)$ и $g(t)$, заключаем, что для каждой точки $u \in U$ условия (6) являются необходимьпи и достаточными для вьполнения критерия потенциальности (7) для оператора (2), а следовательно, и для системы (1). 
ЗАмечАниЕ 1 . Для потенциального оператора $N$ его потенциал $f_{N}$ находится по формуле [8, с. 66]

$$
f_{N}[u]=f_{N}\left[u_{0}\right]+\int_{0}^{1}\left\langle N\left(u_{0}+s\left(u-u_{0}\right)\right), u-u_{0}\right\rangle d s
$$

где $u_{0}$ - фиксированный элемент из $D(N)$.

ЗАмЕчАнИЕ 2. Из доказанной теоремы легко сделать вывод, что операторы, соответствующие дифференциально-разностньм уравнениям запаздывающего типа, не могут быть потенциальными относительно билинейной формы (5).

2. Система первого порядка. Для системы первого порядка

$$
F_{i}\left(t, u(t+\vartheta \tau), u^{\prime}(t+\vartheta \tau)\right)=0
$$

условия потенциальности Гельмгольца (6) имеют вид

$$
\begin{gathered}
{\left[\frac{\partial F_{i}}{\partial u_{j}(t-\tau)}-\frac{d}{d t} \frac{\partial F_{i}}{\partial u_{j}^{\prime}(t-\tau)}\right]_{t \rightarrow t+\tau}=\frac{\partial F_{j}}{\partial u_{i}(t+\tau)}} \\
\frac{\partial F_{i}}{\partial u_{j}(t)}-\frac{d}{d t} \frac{\partial F_{i}}{\partial u_{j}^{\prime}(t)}=\frac{\partial F_{j}}{\partial u_{i}(t)} \\
{\left[\frac{\partial F_{i}}{\partial u_{j}(t+\tau)}-\frac{d}{d t} \frac{\partial F_{i}}{\partial u_{j}^{\prime}(t+\tau)}\right]_{t \rightarrow t-\tau}=\frac{\partial F_{j}}{\partial u_{i}(t-\tau)},} \\
{\left[\frac{\partial F_{i}}{\partial u_{j}^{\prime}(t-\tau)}\right]_{t \rightarrow t+\tau}+\frac{\partial F_{j}}{\partial u_{i}^{\prime}(t+\tau)}=0} \\
{\left[\frac{\partial F_{i}}{\partial u_{j}^{\prime}(t)}+\frac{\partial F_{j}}{\partial u_{i}^{\prime}(t)}=0,\right.} \\
{\left[\frac{\partial F_{i}}{\partial u_{j}^{\prime}(t+\tau)}\right]_{t \rightarrow t-\tau}+\frac{\partial F_{j}}{\partial u_{i}^{\prime}(t-\tau)}=0}
\end{gathered}
$$

где $i, j=1, \ldots, n$.

Из условий (12)-(14) вытекают соотношения

$$
\begin{gathered}
\frac{\partial^{2} F_{i}}{\partial u_{l}^{\prime}(t-\tau) \partial u_{j}^{\prime}(t-\tau)}=0, \quad \frac{\partial^{2} F_{i}}{\partial u_{l}^{\prime}(t) \partial u_{j}^{\prime}(t)}=0, \quad \frac{\partial^{2} F_{i}}{\partial u_{l}^{\prime}(t+\tau) \partial u_{j}^{\prime}(t+\tau)}=0, \\
i, j, l=1, \ldots, n,
\end{gathered}
$$

для вьполнения которых необходимо и достаточно, чтобы функции $F_{i}(11)$ зависели от $u_{j}^{\prime}(t-\tau), u_{j}^{\prime}(t)$ и $u_{j}^{\prime}(t+\tau)$ линейно, т.е. чтобы система $(11)$ имела вид

$$
F_{i} \equiv A_{i}^{k} u_{k}^{\prime}(t-\tau)+B_{i}^{k} u_{k}^{\prime}(t)+C_{i}^{k} u_{k}^{\prime}(t+\tau)+P_{i}=0
$$

где функции $A_{i}^{k}, B_{i}^{k}, C_{i}^{k}, P_{i}$ зависят от переменных $t, u_{j}(t-\tau), u_{j}(t), u_{j}(t+\tau), i, j, k=$ $1, \ldots, n$.

Применяя условия (12)-(15) к левым частям системы (16), получим следующий результат. 
ТЕорема 2. Необходимыми и достаточными условиями потенииальности оператора, соответствующего системе (16), являются условия

$$
\begin{aligned}
& \frac{\partial A_{j}^{k}}{\partial u_{i}(t+\tau)}=0 \\
& {\left[\frac{\partial A_{i}^{k}}{\partial u_{j}(t-\tau)}-\frac{\partial A_{i}^{j}}{\partial u_{k}(t-\tau)}\right]_{t \rightarrow t+\tau}=\frac{\partial B_{j}^{k}}{\partial u_{i}(t+\tau)}} \\
& {\left[\frac{\partial B_{i}^{k}}{\partial u_{j}(t-\tau)}-\frac{\partial A_{i}^{j}}{\partial u_{k}(t)}\right]_{t \rightarrow t+\tau}=\frac{\partial C_{j}^{k}}{\partial u_{i}(t+\tau)}} \\
& {\left[\frac{\partial P_{i}}{\partial u_{j}(t-\tau)}-\frac{\partial A_{i}^{j}}{\partial t}\right]_{t \rightarrow t+\tau}=\frac{\partial P_{j}}{\partial u_{i}(t+\tau)},} \\
& \frac{\partial A_{i}^{k}}{\partial u_{j}(t)}-\frac{\partial B_{i}^{j}}{\partial u_{k}(t-\tau)}=\frac{\partial A_{j}^{k}}{\partial u_{i}(t)} \\
& \frac{\partial B_{i}^{k}}{\partial u_{j}(t)}-\frac{\partial B_{i}^{j}}{\partial u_{k}(t)}=\frac{\partial B_{j}^{k}}{\partial u_{i}(t)} \\
& \frac{\partial C_{i}^{k}}{\partial u_{j}(t)}-\frac{\partial B_{i}^{j}}{\partial u_{k}(t+\tau)}=\frac{\partial C_{j}^{k}}{\partial u_{i}(t)} \\
& \frac{\partial P_{i}}{\partial u_{j}(t)}-\frac{\partial B_{i}^{j}}{\partial t}=\frac{\partial P_{j}}{\partial u_{i}(t)} \\
& {\left[\frac{\partial B_{i}^{k}}{\partial u_{i}(t+\tau)}-\frac{\partial C_{i}^{j}}{\partial u_{k}(t)}\right]_{t \rightarrow t-\tau}=\frac{\partial A_{j}^{k}}{\partial u_{i}(t-\tau)},} \\
& {\left[\frac{\partial C_{i}^{k}}{\partial u_{j}(t+\tau)}-\frac{\partial C_{i}^{j}}{\partial u_{k}(t+\tau)}\right]_{t \rightarrow t-\tau}=\frac{\partial B_{j}^{k}}{\partial u_{i}(t-\tau)}} \\
& \frac{\partial C_{j}^{k}}{\partial u_{i}(t-\tau)}=0 \text {, } \\
& {\left[\frac{\partial P_{i}}{\partial u_{j}(t+\tau)}-\frac{\partial C_{i}^{j}}{\partial t}\right]_{t \rightarrow t-\tau}=\frac{\partial P_{j}}{\partial u_{i}(t-\tau)},} \\
& {\left[A_{i}^{k}\right]_{t \rightarrow t+\tau}+C_{k}^{j}=0,} \\
& B_{i}^{k}+B_{k}^{i}=0, \\
& {\left[C_{i}^{k}\right]_{t \rightarrow t-\tau}+A_{k}^{j}=0 \text {; }}
\end{aligned}
$$

здесь $i, j, k=1, \ldots, n$.

Отметим, что условия (17), (18) получены из равенства (12), условия (19) - из равенства (13), условия (20)-(22) - из равенства (14), а условия (23)-(25) - из соответствующих равенств (15). Кроме того, из условия (17) следует, что функции $A_{i}^{k}$ не зависят от $u_{j}(t+\tau)$, а из условия $(21)$ вытекает, что $C_{i}^{k}$ не зависят от $u_{j}(t-\tau)$. Наконец, условия (23) и (25) эквивалентны.

В заключение заметим, что теории вариационных задач для функционалов с отклоняющимся аргументом и связанных с ними краевых задач посвящены работы [10]-[14]. 
Автор благодарен В. М. Савчину за конструктивное обсуждение заметки и полезные замечания.

\section{СПИСОК ЦИТИРОВАННОЙ ЛИТЕРАТУРЫ}

[1] Helmholtz H. Über die Physikalische Bedeutung des Prinzips der Kleinsten Wirkung // J. Reine Andew. Math. 1887. V. 100. P. 137-166.

[2] Гельмгольц $\Gamma$. О физическом значении принципа наименьшего действия // Вариационные принципы механики / ред. Л. С. Полак. М., 1959. С. 430-459.

[3] Havas P. The connection between conservation laws and invariance groups: folklore, fiction, and fact // Acta Phys. Austriaca. 1973. V. 38. № 2. P. 145-167.

[4] Santilli R. M. Foundations of Theoretical Mechanics. The Inverse Probleme in Newtonian Mechanics. New York: Springer, 1978.

[5] Obadeanu V., Marinca V. Le probléme inverse dans la mécanique newtonienne (théorème analytique fondamental) // Sem. Mech. Univ. Timisoara. 1986. V. 1. P. 1-32.

[6] Филиппов В. М., Савчин В. М., Шорохов С. Г. Вариационные принципы для непотенциальных операторов. Итоги науки и техн. Соврем. пробл. матем. Фундамент. направления. Т. 40. М.: ВИНИТИ, 1992.

[7] Филиппов В. М. Вариационные принципы для непотенциальных операторов. М.: Изд-во Ун-та дружбы народов, 1985.

[8] Савчин В. М. Математические методы механики бесконечномерных непотенциальных систем. М.: Изд-во Ун-та дружбы народов, 1991.

[9] Вайнберг М. М. Функциональный анализ. М.: Просвещение, 1979.

[10] Эльсгольц Л.Э. Вариационные задачи с запаздывающим аргументом // УМН. 1957. Т. 12. № 1. С. $57-58$.

[11] Каменский Г. А. Вариационные и краевые задачи с отклоняющимся аргументом // Дифференц. уравнения. 1970. Т. 6. № 8. С. 1349-1358.

[12] Каменский Г. А. О вариационном методе решения краевых задач для некоторых линейных дифференциальных уравнений с отклоняющимся аргументом // Дифференц. уравнения. 1977. T. 13. № 7. C. $1185-1191$.

[13] Каменский Г. А., Скубачевский А. Л. Экстремумы функционалов с отклоняющимися аргументами. М.: Изд-во МАИ, 1979.

[14] Каменский Г. А., Скубачевский А. Л. Линейные краевые задачи для дифференциально-разностных уравнений. М.: Изд-во МАИ, 1992.

Российский университет дружбы народов, г. Москва 